\title{
Molecule Formation in External Galaxies
}

\author{
T J MILLAR \\ Department of Mathematics, UMIST, PO Box 88, Manchester M60 1QD, UK \\ and \\ E HERBST \\ Physics Department, Ohio State University, 174 W. 18th Ave, Columbus, Ohio \\ 43210, USA
}

September 9, 1991

\begin{abstract}
We discuss the parameters needed to model chemistry in extragalactic clouds. While density and temperature can be constrained by multiline observations, molecular abundances may be severely affected by the adopted elemental abundances. While the observations of the Magelllanic Clouds can be reasonably interpreted in terms of dark cloud models, molecular gas in starburst galaxies could well be dominated by photoeffects. The detection of deuterium in extragalactic molecules would provide a valuable diagnostic.
\end{abstract}

\section{Introduction}

At the time of writing, August 1991, 23 molecules have been detected in external galaxies (see Henkel, this volume). Most molecules have been found in the starburst galaxies NGC 253 and M82, although a significant number have been detected in the metal-poor Large Magellanic Cloud (LMC). Derived molecular abundances from distant galaxies are beam averages over a very large area. From observations of molecular clouds in our own Galaxy, we know that structure exists on all accessible scales and that certain of these structures, for example hot molecular cores, have distinct chemical compositions and abundances from those typical of quiescent molecular clouds. For this reason, the few chemical models applied to external galaxies have been fairly simplistic, although they may be useful in delineating the main synthetic routes to certain species. On the other hand, models of the nearby Magellanic Clouds may be tested by observations with reasonable spatial resolution. Another advantage of studying the Magellanic Clouds is that we have a certain amount of information on stellar properties and on the dust particles which have been studied from ultraviolet to infrared wavelengths.

\section{Chemical Parameters}

In any calculation of molecular abundances, a number of parameters must be specified. We discuss these in turn.

Input densities are usually derived from multi-transition observational studies of polar species such as $\mathrm{CS}$ and $\mathrm{HC}_{3} \mathrm{~N}$. Density variations are obvious. Henkel et al. (1991) have derived densities in the central region of M82 
varying from $\sim 10^{5} \mathrm{~cm}^{-3}$ in the NE molecular 'hotspot' to $\sim 10^{4} \mathrm{~cm}^{-3}$ in the central hotspot, to $>10^{6} \mathrm{~cm}^{-3}$ in the SW hotspot, where $2 \mathrm{~cm} \mathrm{H}_{2} \mathrm{CO}$ emission is observed. Similar variations in density have also been derived for NGC 253 (Mauersberger et al. 1990).

Temperatures are derived in several ways. Vibrationally excited $\mathrm{H}_{2}$ which traces hot molecular gas at $\sim 1000-2000 \mathrm{~K}$ is observed in many galaxies, particularly in starbursts, and may trace the interaction of a flow from the nucleus with surrounding molecular clouds. The molecular observations give a variety of temperatures, typically $20-40 \mathrm{~K}$, although $\mathrm{HC}_{3} \mathrm{~N}$ in $\mathrm{NGC} 253$ indicates that the highest density gas, $\sim 10^{5} \mathrm{~cm}^{-3}$, has a kinetic temperature greater than $60 \mathrm{~K}$. The detection of large abundances of $\mathrm{SiO}$ and $\mathrm{CH}_{3} \mathrm{OH}$ in NGC 253 (Mauersberger and Henkel, 1991) may indicate even higher temperatures since Galactic observations indicate that the abundances of these species are enhanced at high temperature, probably due to the evaporation of mantle material.

There is no reason to believe that the abundances of the elements $\mathrm{C}, \mathrm{N}$, $\mathrm{O}$, etc. are similar in external galaxies. Indeed in the LMC and SMC, the abundances of $\mathrm{C}, \mathrm{N}$ and $\mathrm{O}$ are known to be much less in HII regions than in Orion. For example in the SMC, carbon and nitrogen are reduced by factors of 16 and 14 respectively (see Bel et al., 1986; Barbuy et al., 1991). As we shall discuss below, molecular abundances are, in some cases, extremely sensitive to the adopted elemental abundances.

The dust-to-gas ratio is important because it is related to the dust crosssection which is directly related to the extinction caused by the dust and because the grain surface area affects the rate at which $\mathrm{H}_{2}$ may form.

Molecular clouds can experience UV fluxes much different from those in the solar neighbourhood. The UV flux has a direct effect on photodissociation and photoionisation rates as well as on, through grain photoelectric emission and photoionisation, gas temperatures. The UV flux may vary dramatically on a small scale in external galaxies and may be very large in starbursts. Maloney and Black (1988) find that in the central $450 \mathrm{pc}$ of M82, the mean UV intensity is at least 250 times the local value. This implies that photon-dominated regions (Sternberg, this volume) may play an important role in determining the chemistry of starburst galaxies.

Cosmic rays provide the source of ionisation which drives the chemistry in dark clouds as well as a low-level flux of UV photons. The resulting ionisation rate is poorly known in our own Galaxy (Lepp, this volume) and even more so in external galaxies. If cosmic rays originate in supernovae, the flux may be large in starbursts. X-ray radiation can also provide a source of ionisation in these galaxies. 


\section{Molecular Abundances in External Galaxies}

\subsection{Carbon Monoxide}

Maloney and Black (1988) studied the $\mathrm{C}^{+} / \mathrm{C} / \mathrm{CO}$ transition for model clouds with elemental abundances, dust-to-gas ratios and UV fields typical of those in the LMC and SMC. Because $\mathrm{H}_{2}$ self-shields efficiently against photodissociation, they found that its distribution with depth into the cloud was relatively unaffected by changing the parameters. On the other hand, they found the $\mathrm{CO}$ distribution to differ dramatically because $\mathrm{CO}$ self-shields less efficiently than $\mathrm{H}_{2}$. At the centre of a Galactic cloud, $99 \%$ of the carbon was in CO, for the LMC 5\% and for the SMC $1 \%$. This argues that for CO depthdependent calculations need always to be carried out. For other molecules, this may not be so essential, at least in the cases of the LMC and SMC, since more complex molecules will probably form only in regions in which $\mathrm{CO}$ is abundant.

\subsection{Molecules in the LMC Dark Clouds}

Millar and Herbst (1990) calculated pseudo-time-dependent models for dark clouds in the LMC and SMC and discussed the variation of abundances with adopted parameters. Since then, Johansson (1991) has published molecular abundances derived from the SEST spectral scan of Cloud 2 associated with the HII region N159, just south of 30 Dor in the LMC. We have updated our earlier calculations to include sulphur chemistry and to include ion-dipolar rate coefficients. A comparison of molecular abundances, relative to $\mathrm{CO}$, is given in Table I. Note that the uncertainties in the derived abundances are large (Johansson, 1991) while the time-dependent behaviour of the calculated abundances for some species, particularly SO, HCN and HNC, is severe. The calculations were performed for $\mathrm{n}\left(\mathrm{H}_{2}\right)=10^{4} \mathrm{~cm}^{-3}, \mathrm{~T}=10 \mathrm{~K}$ and $\mathrm{O}, \mathrm{C}$ and $\mathrm{N}$ depleted by factors of $2.8,4.8$ and 12.5 below Galactic dark cloud values, respectively (Millar and Herbst, 1990). While one should not take the agreement between theory and observation too seriously, the agreement may indicate that such crude modelling is worthwhile. If so, this can be tested by observations of the SMC. Millar and Herbst (1990) show that certain molecular abundances should depend not only on the $\mathrm{C} / \mathrm{O}$ ratio but also on the difference $\mathrm{O}-\mathrm{C}$. They find that in their SMC model, which has a carbon abundance only four percent of the Galactic value, the $\mathrm{C}_{2} \mathrm{H}$ fractional abundance is similar to that in Galactic dark clouds, since the abundance of atomic oxygen, which destroys $\mathrm{C}_{2} \mathrm{H}$, is smallest in the SMC model.

Millar and Herbst (1990) found that nitrogen-bearing molecules have a complex behaviour in their various model calculations, in the main because $\mathrm{N}_{2}$ and $\mathrm{NH}_{3}$ formation depend on the abundance of $\mathrm{OH}$, which itself depends 
on the amount of oxygen not tied up in $\mathrm{CO}$, as well as on the atomic oxygen abundance.

Pineau des Forêts et al. (1991) have calculated molecular abundances in dark cloud models in which they systematically vary the depletions of carbon, nitrogen and oxygen, $\delta_{C N O}$, from 1 - 1000 times less than solar. They found, as did Millar and Herbst (1990), that the CO abundance follows that of elemental carbon. In addition, the fractional abundances of species destroyed by atoms, thus principally radicals, tend to increase as depletion increases because of the decreasing abundances of atoms, so that at the largest depletions, the abundance of $\mathrm{OH}$ is larger than that of $\mathrm{CO}$. The abundance of $\mathrm{NH}_{3}$ is remarkably constant in these models with a value which varies by less than an order of magnitude and is close to $10^{-8}$ at both $\delta=1$ and $\delta=1000$. Thus, although $\mathrm{NH}_{3}$ contains only $\sim 10^{-4}$ of the total nitrogen abundance for solar abundances it contains ten percent at $\delta_{C N O}=$ 1000 .

\section{Conclusions}

The chemical models presently developed for interpreting extragalactic observations are rather crude, as indeed is the spatial resolution available in most observations. Abundances in metal poor, dwarf galaxies such as the Magellanic Clouds, appear to be in reasonable agreement with the results of dark cloud models. No models have, as yet, been developed for starburst galaxies. The molecular gas in these galaxies appears to exist in warm, dense clouds - denser than those at the Galactic Centre - bathed by intense radiation fields. Although the molecules detected in NGC 253 and M82, with the exception of $\mathrm{SiO}$ in NGC 253, are also found in Galactic dark clouds, the evidence for a significant dark cloud chemistry has yet to be found. Photon-dominated regions may dominate the chemical evolution of these galaxies.

Finally it is worth stating that a prime observation would be the detection of deuterium in external galaxies. This would give not only important cosmological information but also help delineate the molecule formation process.

\section{References}

BARBUY, B, SPITE, M, SPITE, F and MILONE, A: 1991, Astron. Astrophys. 247, 15 BEL, N, VIALA, Y P and GUIDI, I: 1986, Astron. Astrophys. 160, 301

HENKEL, C, BAAN, W A and MAUERSBERGER, R: 1991, Astron. Astrophys. Rev. 3, 47

JOHANSSON, L E B: 1991, in F COMBES and F CASOLI, ed(s)., Dynamics of Galaxies and Their Molecular Cloud Distributions, Kluwer: Dordrecht, p.1

MALONEY, $\mathrm{P}$ and BLACK, J H: 1988, Ap. J. 325, 389

MAUERSBERGER, R and HENKEL, C: 1991, Astron. Astrophys. 245, 457 
MAUERSBerger, R, Henkel, C and SAGE, L J: 1990, Astron. Astrophys. 236, 63 MILlAR, T J and HERBST, E: 1990, Mon. Not. R. astr. Soc. 242, 92

PINEAU DES FORETS, G, FLOWER, D R and MILLAR, T J: 1991, Mon. Not. R. astr. Soc. , in press

\section{TABLE I}

A comparison of molecular abundances, $\log \mathrm{N}_{C O} / \mathrm{N}_{X}$, in the LMC (Johansson, 1991) with theory. The model parameters are taken from Millar and Herbst (1990). Ion-dipole rate coefficients are adopted and the calculated results are given for the period (1-3.16) $10^{5} \mathrm{yr}$.

$\begin{array}{ccc}\text { Species } & \text { Observed } & \text { Calculated } \\ & & \\ \mathrm{CS} & 1.7-4.5 & 3.2-3.3 \\ \mathrm{CN} & 2.9-4.5 & 3.6-4.8 \\ \mathrm{SO} & 2.1-3.4 & 4.5-3.1 \\ \mathrm{HCO}+ & 2.7-4.2 & 3.9 \\ \mathrm{HCN} & 3.0-4.5 & 4.3-5.4 \\ \mathrm{HNC} & 3.4-4.9 & 4.2-5.4 \\ \mathrm{C}_{2} \mathrm{H} & 2.1-3.6 & 2.7-2.9 \\ \mathrm{H}_{2} \mathrm{CO} & 2.1-4.3 & 2.2-3.0 \\ \mathrm{C}_{3} \mathrm{H}_{2} & 2.6-4.6 & 3.5-3.8\end{array}$




\section{QUESTIONS AND ANSWERS}

M.Guelin: It seems that in your comparisons you should rather speak of molecular column densities (or even integrated line intensities) rather than molecular abundances. Those are far from being known in external galaxies.

T.J.Millar: The determination of column densities from model calculations can be performed if a cloud model is adopted. The problem is that model clouds (spheres, planeparallel slabs) are not at all like real interstellar clouds. Indeed, the poor spatial resolution of observations in external galaxies implies that the observations are averaging over many types of clouds. One thing which might force the theoreticians to calculate line intensities and/or line profiles would be if the observers stopped tabulating fractional abundances!

J.M.Greenberg: I could not understand how $99 \%$ of carbon could be in CO in the center of galactic molecular clouds (from Maloney \& Black). What about the dust which accounts for at least $50 \%$ of the carbon or probably more?

T.J.Millar: Maloney \& Black refer to the gas phase distribution of carbon, not to that in the solid state.

J.M.Greenberg: Getting back to the presence of $99 \%$ of $\mathrm{C}$ in $\mathrm{CO}$ is gas: How about the accretion of $\mathrm{CO}$ on the dust? If $n_{\mathrm{H}_{2}} \simeq 10^{4}$ and we use a small dust/gas ratio then, since $\tau_{a c}$ (accretion time scale) is $\sim 2 \times 10^{9} /\left(2 n_{\mathrm{H}_{2}}\right) \approx 10^{5}$ years, I would expect about $1 / 2 n_{C O}$ on dust and not in gas; i.e. only $50 \%$ of free $\mathrm{C}$ would be in gas $\mathrm{CO}$, unless dust is pretty warm in the cloud certainly which seems exceptional.

T.J.Millar: Maloney and Black performed calculations for $n_{H}=10^{3} \mathrm{~cm}^{-3}$. Also, since the dust-to-gas ratios in the LMC and the SMC are known to be less than the Galactic value, the accretion time-scales in their model clouds are much longer than $10^{6} \mathrm{yr}$, and the gas-grain interaction is therefore negligible.

E.F.van Dishoeck: (comment) The calculations by Maloney \& Black on the $C^{+} \rightarrow C O$ conversion in metal-poor systems stress the importance of depth-dependent models and the comparison of column densities rather than local densities with observations. The assumed size of the cloud is crucial in this respect.

T.J.Millar: This is indeed true in a system such as the SMC, with very low metallicity. It is perhaps not so critical for the LMC - unless one is modelling CO - because the many molecules seen in this galaxy, almost certainty arise from well-shielded regions and not from extended clouds edges. 